\title{
Fabrication of Graphene Nanoplates Modified with Nickel Nanoparticles for Reinforcing Copper Matrix Composites
}

\author{
Tielong Han ${ }^{1}$ Jiajun $\mathrm{Li}^{1} \cdot$ Naiqin Zhao ${ }^{1,3} \cdot$ Chunnian $\mathrm{He}^{1,2,3,4}$
}

Received: 13 September 2019 / Revised: 22 October 2019 / Published online: 14 January 2020

(c) The Chinese Society for Metals (CSM) and Springer-Verlag GmbH Germany, part of Springer Nature 2020

\begin{abstract}
In order to improve the interface wettability as well as the interfacial bonding between graphene and copper matrix, in this work, graphene nanoplates modified with nickel nanoparticles (Ni-GNPs) were synthesized using a one-step method based on spray-drying and chemical vapor deposition. Thereafter, $0.33 \mathrm{wt} \% \mathrm{Ni-GNPs}$ were introduced into copper matrix composite by the molecular-level mixing method, leading to further enhancement of $90 \%$ in yield strength. This is attributed to the presence of Ni-GNPs, which provided high resistance to matrix against deformation. In addition, with the modification of nickel at the interface, the wettability and interfacial bonding between graphene nanoplates and copper matrix were improved, which enhanced the load transfer then. Furthermore, the microstructures and strengthening mechanisms were investigated and discussed meanwhile.
\end{abstract}

Keywords Carbon materials $\cdot$ Composite materials $\cdot$ Chemical vapor deposition $\cdot$ Nanocomposites

\section{Introduction}

Known as an ideal reinforcement for metal matrix composites (MMCs), graphene has drawn great attention due to its excellent mechanical and electrical properties in the past decades [1]. Moreover, graphene possesses larger specific surface area compared with carbon nanotubes, which results

Available online at http://link.springer.com/journal/40195.

Electronic supplementary material The online version of this article (https://doi.org/10.1007/s40195-020-00999-0) contains supplementary material, which is available to authorized users.

Chunnian $\mathrm{He}$

cnhe08@tju.edu.cn

1 School of Materials Science and Engineering and Tianjin Key Laboratory of Composites and Functional Materials, Tianjin University, Tianjin 300072, China

2 Joint School of National University of Singapore and Tianjin University, International Campus of Tianjin University, Binhai New City, Fuzhou 350207, China

3 Collaborative Innovation Center of Chemical Science and Engineering, Tianjin 300072, China

4 Key Laboratory of Advanced Ceramics and Machining Technology, Ministry of Education, Tianjin University, Tianjin 300072, China in much more effective load transfer and makes it a better reinforcement for composites [2]. Therefore, graphene and its derivatives have been widely used for the reinforcing of all kinds of MMCs with improved mechanical and functional performance in recent years [2-6]. For instance, graphene nanoplates (GNPs)-reinforced aluminum matrix composites prepared by Shin et al. [2] show superhigh tensile strength. Also, using a disintegrated meld deposition method, 3 wt\% GNPs was dispersed into the AZ61 magnesium matrix and resulted in significant improvement in room temperature mechanical properties [3]. And improved compressive strength was achieved by the introduction of graphene in the work of Yang et al. [4]. Furthermore, the thermal and electrical conductivity as well as the mechanical properties of copper could also be enhanced by introducing graphene in the matrix [5-7]. However, the poor wettability between graphene and copper usually results in a poor cohesion strength and negligible load transfer at copper/graphene interface [8], so that the potential of graphene could not be fully realized. Thus, the post-treatment process was conducted to improve the interfacial bonding between graphene and copper matrix in the work of Saboori et al. [9] and improved densification and mechanical properties were achieved then. Furthermore, it is found that the $\mathrm{Cr}_{7} \mathrm{C}_{3}$ layers formed at the graphene-CuCr interface could dramatically improve the interfacial bonding of the composites, also [8]. 
Tang et al. [10] and Zhang and Zhan [11] planted $\mathrm{Ni}$ and $\mathrm{Cu}$ nanoparticles on graphene and then improved wettability and reinforcing effect were obtained. These all inspire us that appropriate interfacial modification could be significant in graphene-reinforced copper matrix composites.

However, the secondary processed chemical plating is usually required for the synthesis of metal modified graphene $[10,11]$, which is really a tedious process and adverse to the tight integration of graphene and metal plating. In addition, the graphene used in most of the studies was prepared by the Hummers' method or the mechanical exfoliation method, which is usually with high cost and low productivity. By contrast, a one-step bottom-up strategy we proposed recently [12] could be a good choice for the synthesis of nickel nanoparticles modified GNPs aiming at a high production efficiency and tight interfacial bonding between graphene and nickel nanoparticles. Moreover, this method allows the synthesis and modification of GNPs in one step, which is more facile, time-saving, low-cost, and suitable for mass production.

Since the strengthening effect of the GNPs synthesized by this bottom-up method has been verified in aluminum matrix [12], it is expected to play a strong enhancement effect in copper matrix. Thus, in this study Ni-modified graphene nanoplates (Ni-GNPs) were synthesized with the bottom-up method and then introduced into $\mathrm{Cu}$ matrix by the molecular-level mixing (MLM) method [13] followed by hot-pressing. Notably, nickel salt was changed in this work in order to expand the applicability of the bottom-up method. As a result, copper matrix composite reinforced with $0.33 \mathrm{wt} \%$ Ni-GNPs shows obviously improvement in mechanical properties, demonstrating that the Ni-GNP prepared by the novel method is also an effective reinforcement for copper. Meanwhile, the microstructures of the composite were carefully characterized and the strengthening mechanisms were analyzed and discussed.

\section{Experimental}

\subsection{Sample Preparation}

For the synthesis of Ni-GNPs, the bottom-up strategy based on spray-drying and chemical deposition was used. Nickel chloride, glucose and sodium chloride (99.9\% in purity) with a certain ratio were dissolved in deionized water and then dried by spray-drying. The as-obtained precursors were then heat-treated at $730{ }^{\circ} \mathrm{C}$ in $\mathrm{H}_{2}$ atmosphere for $2 \mathrm{~h}$. NiGNPs powder was obtained after washing the $\mathrm{NaCl}$ away by deionized water. Subsequently, copper matrix composite powders containing $0.33 \mathrm{wt} \%$ of Ni-GNPs were fabricated by MLM method, followed by a hot-press sintering approach at $800{ }^{\circ} \mathrm{C}$ for another an hour. For comparison, pure $\mathrm{Cu}$ and reference sample containing $0.33 \mathrm{wt} \%$ of Ni nanoparticles were also prepared with the same process. (The schematic diagram for the preparation procedure is shown in Fig. S1, and more details about the preparation process are provided in Supporting Information.)

\subsection{Characterization}

Raman spectra were conducted using a Renishaw inVia Raman microscope. The morphology of the samples was observed by a Hitachi S-4800 field emission scanning electron microscopy (SEM). And transmission electron microscopy (TEM) was performed on a JEOL JEM-2100F microscope. In addition, the tensile tests were carried out on a LLOYD EZ20 tensile tester at an initial strain rate of $8.3 \times 10^{-4} \mathrm{~s}^{-1}$.

\section{Results and Discussion}

A self-assembled structure with several stacked $\mathrm{NaCl}$ crystals covered with an ultrathin glucose $/ \mathrm{NiCl}_{2}$ mixture film was obtained after spray-drying, as shown in Fig. 1a. Then during the heat treatment process, glucose was decomposed gradually and served as the carbon source while $\mathrm{NiCl}_{2}$ was reduced into Ni nanoparticles. Thereafter, the amorphous carbon atoms got graphitized gradually under the catalysis of Ni nanoparticles at high temperatures, and Ni-GNPs then formed on the surfaces and interfaces of $\mathrm{NaCl}$ crystals, which can be clearly observed in Fig. 1b. Finally, foliated structural GNPs decorated with Ni particles were obtained after removing $\mathrm{NaCl}$, as shown in Fig. 1c. The structure of Ni-GNPs powder was then further studied by the TEM, as shown in Fig. 1d-f, from which we can find that Ni nanoparticles with diameters of approximately $80 \mathrm{~nm}$ and $10 \mathrm{~nm}$ were anchored on the surface of GNPs uniformly, which is well consistent with the results of SEM. Figure $1 \mathrm{f}$ shows the edge of GNP in a high magnification, where the layer structure of GNP could be observed clearly and the thickness of the synthesized GNPs is less than $10 \mathrm{~nm}$. It is noteworthy that those Ni nanoparticles still combine tightly with the GNPs even after a longtime sonication $(1 \mathrm{~h})$, indicating the strong bonding between Ni and GNPs as well as the high structural stability of Ni-GNPs.

Raman spectra of Ni-GNPs powders and the bulk NiGNPs/Cu composite are shown in Fig. 2a, where peaks around 1340 and $1600 \mathrm{~cm}^{-1}$ corresponding to the $\mathrm{D}$ band and $\mathrm{G}$ band of graphene, respectively, could be found for both materials. Usually, D band represents the structural defects of graphene, while $\mathrm{G}$ band belongs to the typical ordered graphite structure attributed to the vibration of $s p^{2}$-bonded carbon [14]. Therefore, the intensity ratio of $\mathrm{D}$ band to $\mathrm{G}$ band $\left(I_{\mathrm{D}} / I_{\mathrm{G}}\right)$ was often used to estimate the defects 

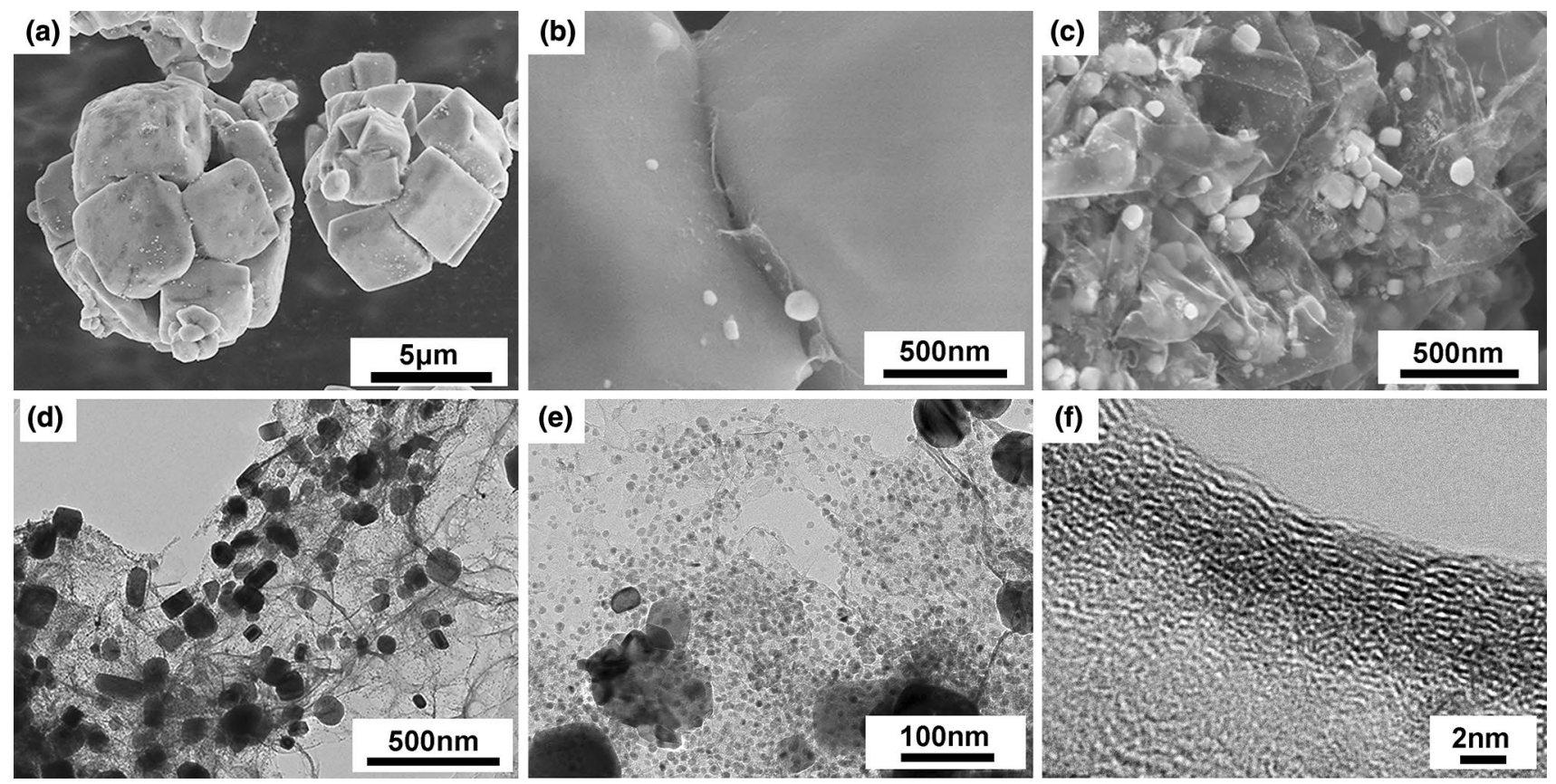

Fig. 1 SEM images of a the heat-treated spray-dried precursors with a self-assembly structure, $\mathbf{b}$ the magnified image of a, $\mathbf{c}$ the obtained NiGNPs powders. $\mathbf{d}-\mathbf{f}$ TEM images of Ni-GNPs powders with different magnifications
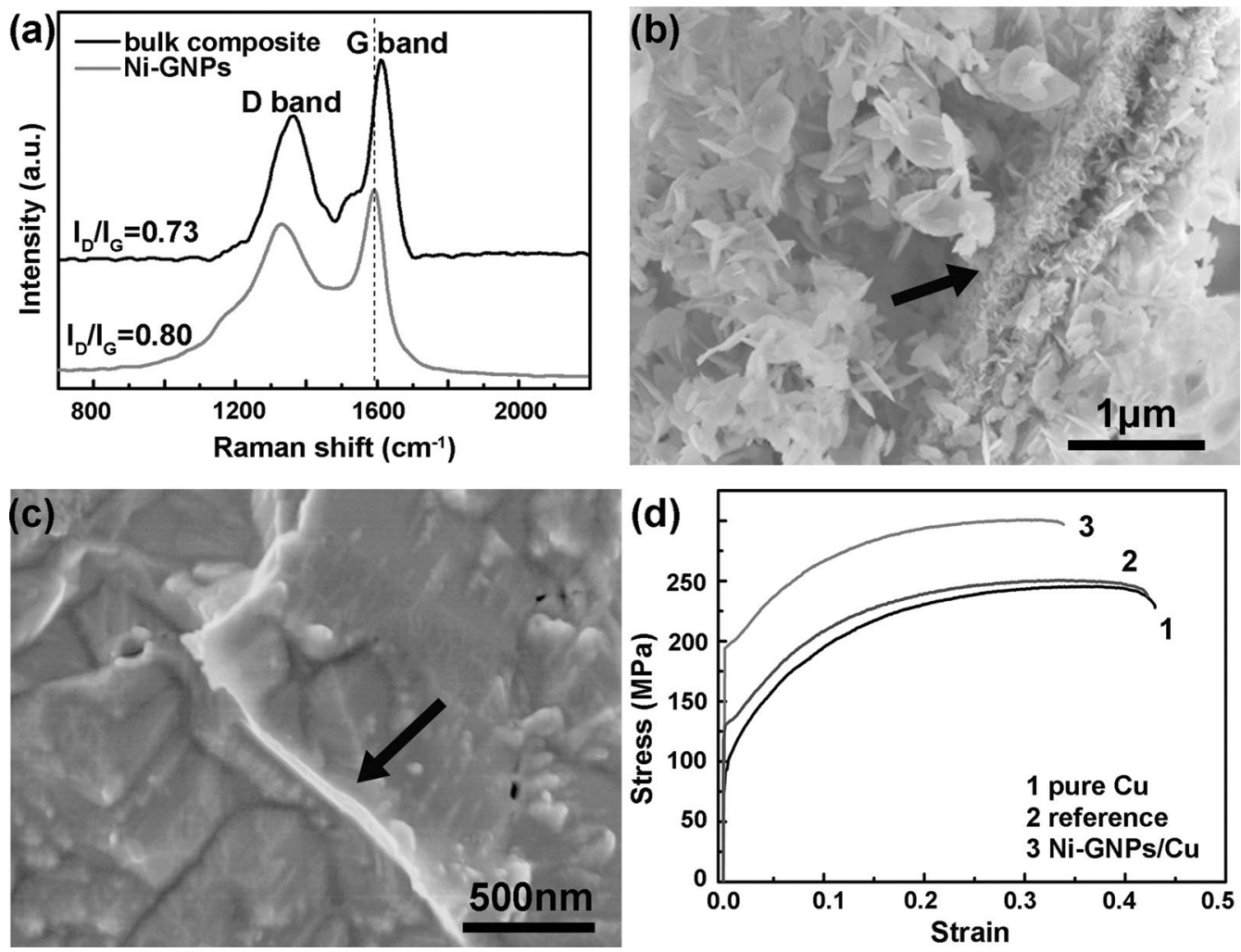

Fig. 2 a Raman spectra of Ni-GNPs powders and bulk composite; SEM image of $\mathbf{b}$ the composite powders and $\mathbf{c}$ the etched surface of the bulk composite; $\mathbf{d}$ the engineering strain-stress curves 
presented in graphene $[10,11]$. As shown in Fig. 2a, the $I_{\mathrm{D}} / I_{\mathrm{G}}$ values of Ni-GNPs powders and bulk composite are approximately 0.80 and 0.73 , respectively, indicating that the number of defects in GNPs was reduced after hot-pressing. This is mainly due to the higher treatment temperature during hot-pressing as well as the secondary catalysis of copper matrix, for $\mathrm{Cu}$ is also a very excellent catalytic substrate and has been widely used for the growth of graphene $[15,16]$. Additionally, it can be found that the G band of GNPs in bulk composite exhibits a red-shift compared with that of $\mathrm{Ni}$-GNPs powders, revealing a significant electronic interactions between GNPs and copper matrix [10, 17], which is beneficial to the interfacial bonding and then contributes to the load transfer effect. Moreover, the SEM image of composite powders obtained after MLM process is shown in Fig. 2b. GNPs were surrounded by $\mathrm{CuO}$ tightly, as marked with a white arrow, indicating good bonding between $\mathrm{CuO}$ and Ni-GNPs. (The confirmation of $\mathrm{CuO}$ is based on the XRD patterns as shown in Fig. S2, Supporting Information.) Furthermore, the GNPs could be observed in the bulk composite without any aggregation after etching the surface with $\mathrm{FeCl}_{3}$ solution (Fig. 2c).

The engineering strain-stress curves are shown in Fig. 2d, from which we can see that the composite exhibited a yield strength of $190 \mathrm{MPa}$, which was improved by $90 \%$ compared with that of pure copper (100 MPa), while the reference sample containing $0.33 \mathrm{wt} \% \mathrm{Ni}$ only showed slight improvement. Therefore, it can be concluded that the significant improvement in strength is mainly caused by the addition of GNPs instead of Ni particles. Moreover, it is worth noting that only $0.33 \mathrm{wt} \%$ of Ni-GNPs was introduced and resulted in a superhigh yield strength, demonstrating a high reinforcing efficiency of Ni-GNPs in copper matrix. To make it clear, the reinforcing efficiency $R$ (defined as $R=\left(\sigma_{\mathrm{c}}-\sigma_{\mathrm{m}}\right) / V \sigma_{\mathrm{m}}$, where $\sigma_{\mathrm{c}}, \sigma_{\mathrm{m}}$ and $V$ are the strength of composite, strength of matrix and the volume percentage of reinforcement) of the Ni-GNPs/Cu composite was calculated and compared with other works [10,11, 13, 16, 18, 19], as listed in Table 1. (Since the amount of GNPs in Ni-GNPs is unknown, a median 50\% was used for the calculation.) As listed in Table 1, all the Ni modified graphene structures show very high $R$ values compared with the unvarnished graphene, indicating the modification of $\mathrm{Ni}$ on the surface of graphene could indeed enhance the interfacial bonding.

The grain size and grain orientation of pure $\mathrm{Cu}$ and $\mathrm{Ni}$ GNPs/Cu composite were determined by the electron backscattered diffraction (as shown in Fig. 3). More than 1000 grains were counted and the statistical graphs of grain size distribution are shown in Fig. $3 \mathrm{c}$ and d. Both show equiaxed grains, while the average grain size of the composite and pure copper is about 2.17 and $7.95 \mu \mathrm{m}$, respectively. Obvious grain refining effect was achieved by introducing Ni-GNPs due to the impeding effect of GNPs to the grain
Table 1 Comparison of the reinforcing efficiency $(R)$ of various reinforcements for copper matrix composites

\begin{tabular}{lrl}
\hline Reinforcements & $R(\%)$ & References \\
\hline Ni-GNPs & 150 & This work \\
Graphene nanosheets-Ni & 94 & Tang et al. [10] \\
Ni-GNPs & 129 & Zhang et al. [11] \\
Reduced graphene oxide & 45 & Hwang el al. [13] \\
In situ grown graphene & 67 & Chen et al. [16] \\
Reduced graphene oxide & 13 & Gao et al. [18] \\
Graphene nanoribbons & 33 & Zhang et al. [19] \\
\hline
\end{tabular}

coarsening during thermal processing [12]. According to the Hall-Petch relation, the finer grains contribute to higher strength for the increased grain boundaries and improved grain boundary strengthening [20].

In addition, the microstructure of the Ni-GNPs/Cu composite was also investigated by TEM. As shown in Fig. 4a, GNPs were marked by white circles while the grain boundaries were marked by white dashes. It is obvious that the GNPs distributed inside the matrix uniformly, which could impede the movement of dislocations and bridge the cracks, leading to an improved strength of the composite [19, 21]. Figure $4 \mathrm{~b}$ shows the high resolution TEM image of the selected area in Fig. 4a. A smooth transition zone could be found, and the interface is free of impurities, voids or gaps, indicating good bonding between GNPs and copper matrix. Since nickel was not observed during the TEM tests, it was further confirmed by the energy-dispersive spectroscopy (EDS), as shown in Fig. 4c and d. By considering the infinite solid solution of copper and nickel, it was believed that $\mathrm{Ni}$ dissolved in $\mathrm{Cu}$ matrix and $\mathrm{Cu}-\mathrm{Ni}$ solid solution was formed near the interface of GNPs and $\mathrm{Cu}$ matrix. The formation of $\mathrm{Cu}-\mathrm{Ni}$ solid solution contributes to reduce the interface energy between GNPs and $\mathrm{Cu}$ and, in turn, strengthens their interfacial bonding [10]. The strong interfacial bonding ensured high load transfer efficiency in composites, which might be one of the reasons for the improved yield strength.

\section{Conclusions}

In summary, Ni-GNPs were synthesized by a one-step bottom-up method using nickel chloride, glucose and $\mathrm{NaCl}$ as the raw materials. The universality of this bottom-up method is expanded and verified. Ni-GNPs fabricated by this method show great strengthening effect in copper matrix composites, which is mainly due to the improved load transfer effect and the grain refine effect. The strength was expected to be further improved by improving the content of Ni-GNPs, and our future work will focus on the optimization of the processing parameters, microstructure of the Ni-GNPs powder, with the 

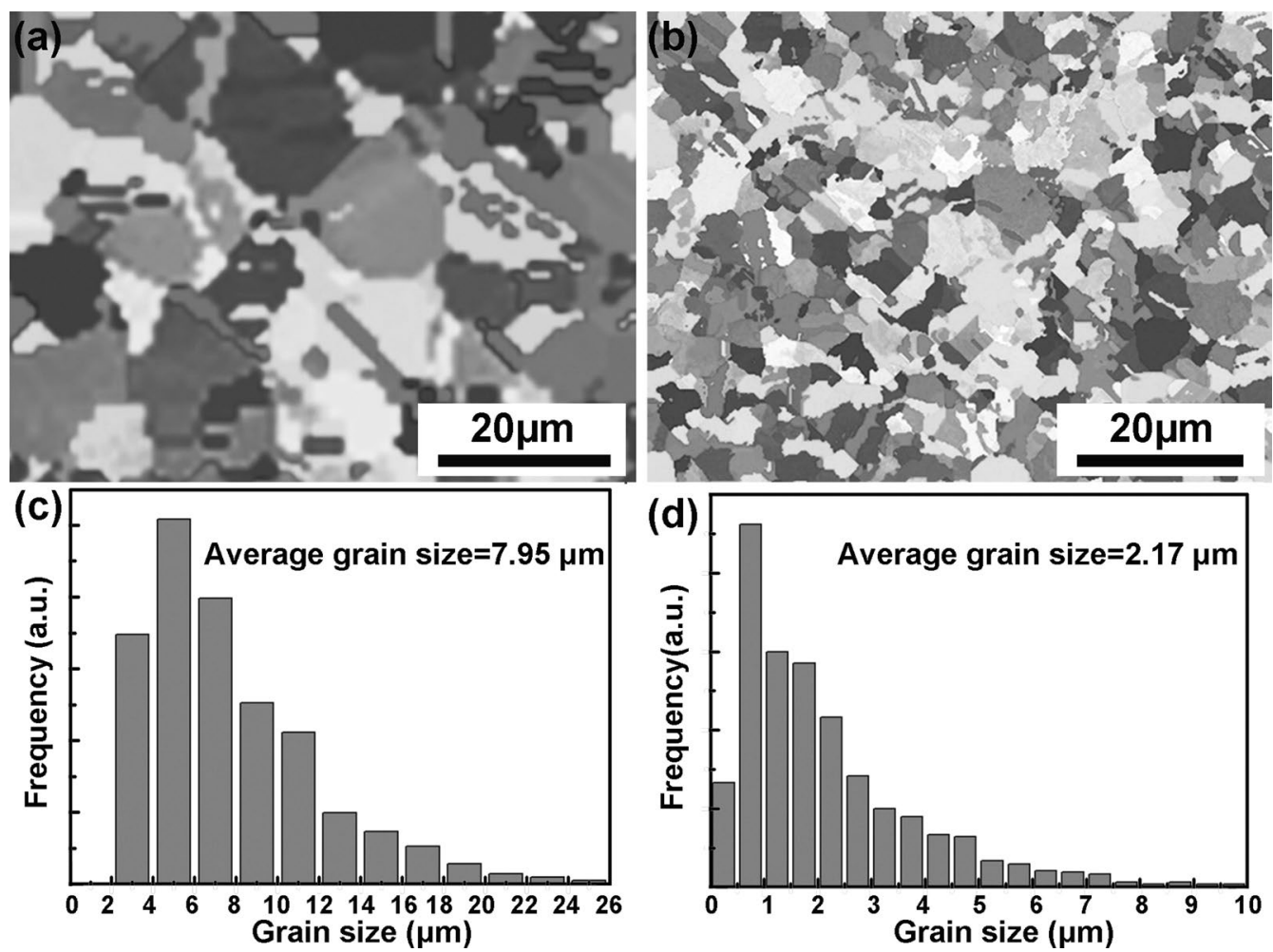

Fig. 3 Orientation imaging microscopy maps of a unreinforced $\mathrm{Cu}$ and $\mathbf{b}$ Ni-GNPs/Cu composite; $\mathbf{c}$ and $\mathbf{d}$ are the corresponding statistical graphs of grain size distribution, respectively
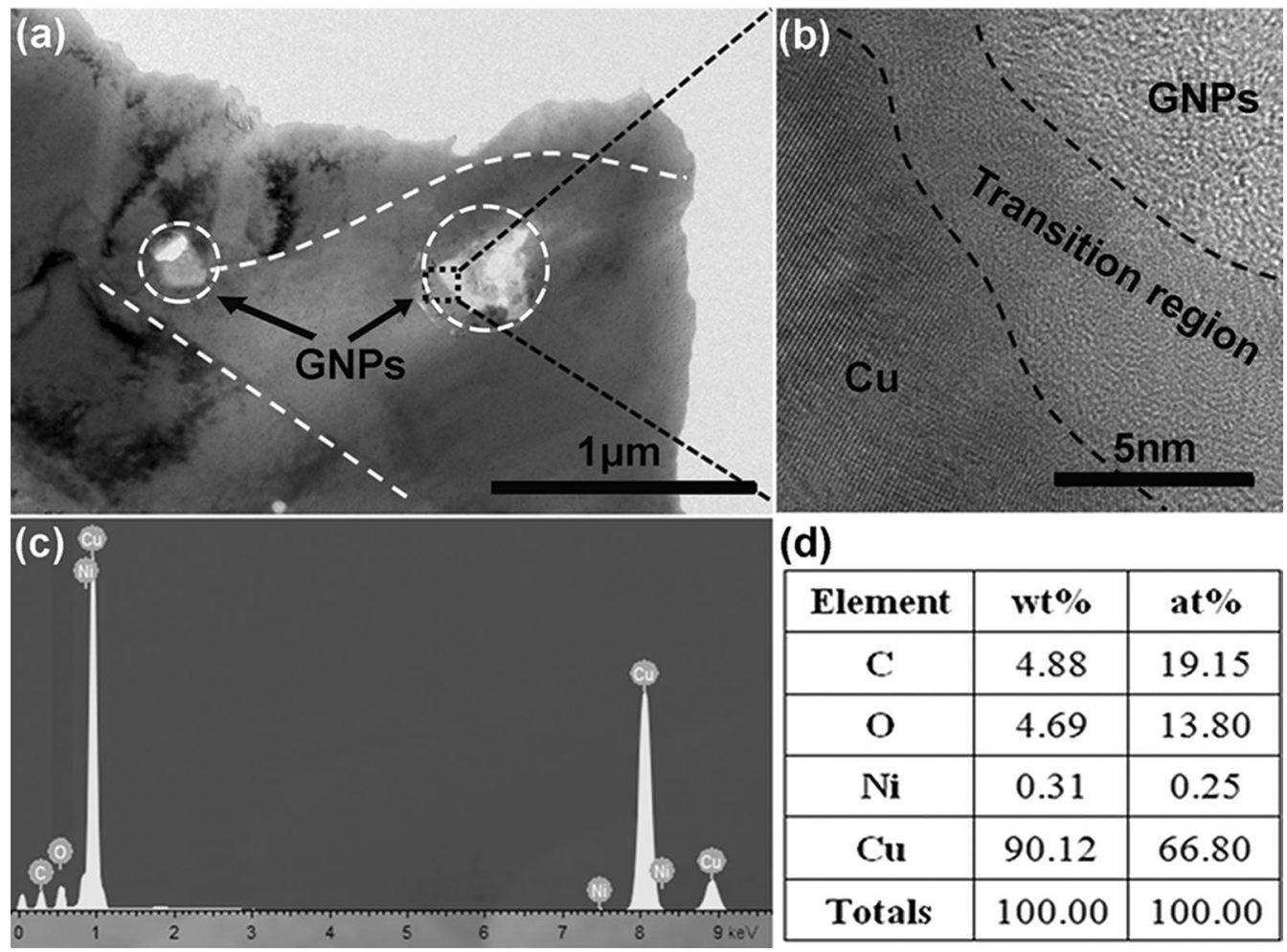

(d)
\begin{tabular}{|c|c|c|}
\hline Element & $\mathbf{w t} \%$ & at\% \\
\hline $\mathrm{C}$ & 4.88 & 19.15 \\
\hline $\mathrm{O}$ & 4.69 & 13.80 \\
\hline $\mathrm{Ni}$ & 0.31 & 0.25 \\
\hline $\mathrm{Cu}$ & 90.12 & 66.80 \\
\hline Totals & 100.00 & 100.00 \\
\hline
\end{tabular}

Fig. 4 a TEM image of the bulk composite; $\mathbf{b}$ TEM image of the selected area in $\mathbf{a} ; \mathbf{c}, \mathbf{d}$ EDS analysis of $\mathbf{b}$ 
aim of gaining better performance. Some other matrix would be utilized, and further explorations on the strengthening mechanisms would be taken.

Acknowledgements The work was financially supported by the National Natural Science Funds for Excellent Young Scholar (Grant No. 51422104), the National Natural Science Foundation of China (Grant Nos. 51531004, 51771130 and 51472177), and the Foundation for the Author of National Excellent Doctoral Dissertation of China (Grant No. 201145).

\section{References}

[1] S. Park, R.S. Ruoff, Nat. Nanotechnol. 4, 217 (2009)

[2] A. Saboori, M. Pavese, C. Badini, P. Fino, Acta Metall. Sin. (Engl. Lett.) 30, 675 (2017)

[3] M. Rashad, F. Pan, D. Lin, M. Asif, Mater. Des. 89, 1242 (2016)

[4] W.Z. Yang, W.M. Huang, Z.F. Wang, F.J. Shang, W. Huang, B.Y. Zhang, Acta Metall. Sin. (Engl. Lett.) 29, 707 (2016)

[5] M. Cao, D.B. Xiong, L. Yang, S. Li, Y. Xie, Q. Guo, Z. Li, H. Adams, J. Gu, T. Fan, X. Zhang, D. Zhang, Adv. Funct. Mater. 29, 1806792 (2019)

[6] K. Chu, X. Wang, F. Wang, Y. Li, D. Huang, H. Liu, W. Ma, F. Liu, H. Zhang, Carbon 127, 102 (2018)
[7] X. Zhang, C. Shi, E. Liu, F. He, L. Ma, Q. Li, J. Li, W. Bacsa, N. Zhao, C. He, Nanoscale 9, 11929 (2017)

[8] K. Chu, F. Wang, Y. Li, X. Wang, D. Huang, H. Zhang, Carbon 133, 127 (2018)

[9] A. Saboori, M. Pavese, C. Badini, P. Fino, Metall. Mater. Trans. A 49, 333 (2018)

[10] Y. Tang, X. Yang, R. Wang, M. Li, Mater. Sci. Eng. A 599, 247 (2014)

[11] D. Zhang, Z. Zhan, J. Alloys Compd. 658, 663 (2016)

[12] T. Han, J. Li, N. Zhao, C. He, Carbon 159, 311-323 (2020)

[13] J. Hwang, T. Yoon, S.H. Jin, J. Lee, T.S. Kim, S.H. Hong, S. Jeon, Adv. Mater. 25, 6274 (2013)

[14] A.C. Ferrari, Solid State Commun. 143, 47 (2007)

[15] J.D. Wood, S.W. Schmucker, A.S. Lyons, E. Pop, J.W. Lyding, Nano Lett. 11, 4547 (2011)

[16] Y. Chen, X. Zhang, E. Liu, C. He, C. Shi, J. Li, P. Nash, N. Zhao, Sci. Rep. 6, 19363 (2016)

[17] A. Rao, P. Eklund, S. Bandow, A. Thess, R.E. Smalley, Nature 388, 257 (1997)

[18] X. Gao, H. Yue, E. Guo, H. Zhang, X. Lin, L. Yao, B. Wang, Powder Technol. 301, 601 (2016)

[19] M. Yang, L. Weng, H. Zhu, T. Fan, D. Zhang, Carbon 118, 250 (2017)

[20] N. Hansen, Scr. Mater. 51, 801 (2004)

[21] Z. Li, L. Zhao, Q. Guo, Z. Li, G. Fan, C. Guo, D. Zhang, Scr. Mater. 131, 67 (2017) 